\title{
RECTIFYING CURVES AND GEODESICS ON A CONE IN THE EUCLIDEAN 3-SPACE
}

\author{
BANG-YEN CHEN
}

In memory of Professor Tadashi Nagano

\begin{abstract}
A twisted curve in the Euclidean 3-space $\mathbb{E}^{3}$ is called a rectifying curve if its position vector field always lie in its rectifying plane. In this article we study geodesics on an arbitrary cone in $\mathbb{E}^{3}$, not necessary a circular one, via rectifying curves. Our main result states that a curve on a cone in $\mathbb{E}^{3}$ is a geodesic if and only if it is either a rectifying curve or an open portion of a ruling. As an application we show that the only planar geodesics in a cone in $\mathbb{E}^{3}$ are portions of rulings.
\end{abstract}

\section{Introduction}

A geodesic on a surface is a curve connecting two nearby points such that any nearby curve with the same endpoints is longer. The study of geodesics on surfaces is one of the most fundamental and important problems in classical differential geometry (cf. $[2,9,8,10$, 11]). The study of geodesics has many applications in mathematics, sciences and as well as in engineerings.

Consider geodesics on a circular cone. If we cut the circular cone along a ruling, then the cone unwraps into a sector of the Euclidean plane, and the geodesics on the cone must yield straight line segments in the sector.

If we try to determine geodesics on a circular cone in $\mathbb{E}^{3}$ analytically, the equations for geodesics are messy in general and chock full of partial derivatives. If we put that approach aside and consider the problem geometrically, then the following facts are well-known: The number of distinct geodesics connecting two points is always finite and it depends essentially only on the angle of the circular cone, plus there will be a few special cases, corresponding to specially chosen boundary conditions. Distinct geodesics connecting the two points will differ by how many times they wrap around the circular cone, and the shortest one will have the smallest angle change.

Received December 19, 2016, accepted January 2, 2017.

2010 Mathematics Subject Classification. 53A04, 53A05, 53C22.

Key words and phrases. Geodesic, cone, circular cone, rectifying curve. 
Next, let us consider a general cone with vertex at $o \in \mathbb{E}^{3}$. For a given curve $\mathbf{y}=\mathbf{y}(t)$ defined on an open interval $I$ lying on the unit sphere $S_{o}^{2}(1)$ centered at $o \in \mathbb{E}^{3}$. Let $C_{\mathbf{y}}$ denote the cone with vertex at o over the curve $\mathbf{y}$. Clearly, $C_{\mathbf{y}}$ can be parametrized as

$$
C_{\mathbf{y}}(t, u)=u \mathbf{y}(t), \quad u \in \mathbf{R}^{+} .
$$

For $t_{0} \in I$, we call the curve $\beta(u)=C_{\mathbf{y}}\left(t_{0}, u\right), u \in \mathbf{R}^{+}$, a ruling (or generating half line). Obviously, the cone $C_{\mathbf{y}}$ is generated by the rulings.

If we choose the curve $\mathbf{y}$ in (1.1) to be a small circle on $S_{o}^{2}(1)$, then the cone is well-known as a circular cone. And if the curve $\mathbf{y}$ is a great circle on $S_{o}^{2}(1)$, the cone is a plane. Clearly, geodesics on a plane are nothing but line segment. Therefore we only consider cones which are not planes throughout this article.

The main purpose of this article is to classify all geodesics on a cone (not necessary a circular one) via rectifying curves. Our main result states that a curve on a cone in $\mathbb{E}^{3}$ is a geodesic if and only if it is either a rectifying curve or an open portion of a ruling. As an application we show that the only planar geodesics in a cone in $\mathbb{E}^{3}$ are portions of rulings.

\section{Rectifying curves}

In differential geometry, most geometers describe a curve as a unit speed curve $\mathbf{x}=\mathbf{x}(s)$ whose position vector field (with reference point $o$ ) is expressed in term of an arc-length parameter $s$. In order to define curvature and torsion of a space curve, one needs the well-known Frenet formulas which can be obtained as follows:

Consider a unit-speed curve $\mathbf{x}: I \rightarrow \mathbb{E}^{3}$, defined on a real interval $I=(\alpha, \beta)$, that has at least four continuous derivatives. Put $\mathbf{t}=\mathbf{x}^{\prime}(s)$. In general, it is possible that $\mathbf{t}^{\prime}(s)=0$ for some $s$; however, we assume that this never happens. Then we can introduce a unique vector field $\mathbf{n}$ and positive function $\kappa$ so that $\mathbf{t}^{\prime}=\kappa \mathbf{n}$. We call $\mathbf{t}^{\prime}$ the curvature vector field, $\mathbf{n}$ the principal normal vector field, and $\kappa$ the curvature of the given curve $\mathbf{x}(t)$. Since $\mathbf{t}$ is a constant length vector field, $\mathbf{n}$ is orthogonal to $\mathbf{t}$. The binormal vector field is defined by $\mathbf{b}=\mathbf{t} \times \mathbf{n}$ which is a unit vector field orthogonal to both $\mathbf{t}$ and $\mathbf{n}$. One defines the torsion $\tau$ of the curve by the equation $\mathbf{b}^{\prime}=-\tau \mathbf{n}$.

The famous Frenet formulas are given by

$$
\left\{\begin{array}{l}
\mathbf{t}^{\prime}=\kappa \mathbf{n}, \\
\mathbf{n}^{\prime}=-\kappa \mathbf{t}+\tau \mathbf{b}, \\
\mathbf{b}^{\prime}=-\tau \mathbf{n} .
\end{array}\right.
$$

At each point of the curve, the planes spanned by $\{\mathbf{t}, \mathbf{n}\},\{\mathbf{t}, \mathbf{b}\}$ and $\{\mathbf{n}, \mathbf{b}\}$ are known as the osculating plane, the rectifying plane, and the normal plane, respectively. 
It is well-known that a curve in $\mathbb{E}^{3}$ lies in a plane if its position vector lies in its osculating plane at each point; and it lies on a sphere if its position vector lies in its normal plane at each point. In view of these basic facts, the author asked the following very simple natural geometric question in [3] (see also [6]):

Question. When does the position vector (with reference point o) of a space curve $\mathbf{x}: I \rightarrow \mathbb{E}^{3}$ always lie in its rectifying plane?

We simply call such a curve a rectifying curve in [3]. It is known that rectifying curves have many interesting properties (see, for instance, $[3,4,5,6,7]$ ).

\section{Main result}

For our main theorem we need the following lemmas.

Lemma 1. Every open portion of a ruling is a geodesic of the cone.

Proof. Assume that $\gamma$ is an open portion of a ruling defined by

$$
\beta(u)=u \mathbf{y}\left(t_{0}\right), u \in(a, b),
$$

where $\mathbf{y}(t)$ is a unit speed curve on the cone. It follows from (3.1) that

$$
\beta^{\prime}(u)=\mathbf{y}\left(t_{0}\right), \beta^{\prime \prime}(u)=0
$$

which implies that $\beta^{\prime}(u)$ is a unit vector field and the geodesic curvature $\kappa_{g}$ of $\gamma$ in the cone vanishes identically. Hence $\gamma$ is a geodesic of the cone.

Lemma 2. Let $\mathbf{y}(t)$ be a unit speed curve on the unit sphere $S_{o}^{2}(1)$ in $\mathbb{E}^{3}$. Then, for each $c \in \mathbf{R}^{+}$, the curve $\mathbf{z}(t)=c \mathbf{y}(t)$ is not a geodesic of the cone $\mathscr{C}$ defined by (1.1).

Proof. Assume that $\mathbf{y}(t)$ is a unit speed curve on $S_{o}^{2}(1)$. For a positive number $c$, consider the curve $\mathbf{z}(t)=c \mathbf{y}(t)$. Let $\kappa_{g}$ denote the geodesic curvature of $\mathbf{z}$. It is well-known that the geodesic curvature $\kappa_{g}$ is an intrinsic invariant (cf., e.g., [8, Proposition 4.4, page 106]). Since the cone is a flat surface and $|\mathbf{z}|=c$, the geodesic curvature $\kappa_{g}$ of $\mathbf{z}$ is equal to $c^{-1} \neq 0$. Consequently, $\mathbf{z}=c \mathbf{y}$ cannot be a geodesic on the cone.

The main result of this article is the following theorem which classifies all geodesics on a cone in a very simple way.

Theorem 1. A curve on a cone $\mathscr{C}$ with vertex at $\mathrm{E} \in \mathbb{E}^{3}$ is a geodesic if and only if it is either a rectifying curve (with reference point o) or it is an open portion of a ruling. 
Proof. Let $\mathscr{C}$ be a cone parametrized by $C_{\mathbf{y}}(t, u)=u \mathbf{y}(t)$, where $\mathbf{y}(t)$ is a curve on the unit sphere $S_{o}^{2}(1)$ centered at $o \in \mathbb{E}^{3}$. Without loss of generality, we may assume that $\mathbf{y}(t)$ is a unit speed curve.

Our goal is to determine all geodesics on the cone $\mathscr{C}$. Now, let $\mathbf{z}$ be a curve on $\mathscr{C}$. If $\mathbf{z}$ is an open portion of a ruling, then it is a geodesic on $\mathscr{C}$ according to Lemma 1 . Hence, from now on, we may assume that $\mathbf{z}$ does not lie in any ruling. Clearly, if $\mathbf{z}$ is a geodesic and if an open portion of $\mathbf{z}$ lies in a ruling, then that portion is a geodesic of the cone. Thus the whole geodesic $\mathbf{z}$ lies in the ruling. Therefore we may assume that no open portion of $\mathbf{z}$ lies in any ruling. Consequently, we may put

$$
\mathbf{z}(t)=\rho(t) \mathbf{y}(t), \quad t \in I,
$$

for some function $\rho(t)$, where $I$ is an open interval.

If $\rho$ is a constant, say $c \neq 0$, on an open subinterval $J \subset I$, then (3.3) becomes $\mathbf{z}(t)=c \mathbf{y}(t)$ on $J$, which is never a geodesic of the cone by Lemma 2. Therefore we may further assume that $\rho^{\prime} \neq 0$. Since $\mathbf{y}(t)$ is a unit speed curve on $S_{o}^{2}(1)$, we find from (3.3) that

$$
\left|\mathbf{z}^{\prime}(t)\right|^{2}=|\rho(t)|^{2}+\left|\rho^{\prime}(t)\right|^{2} .
$$

Let us consider the following integral functional with fixed end points:

$$
L\left(t, \rho, \rho^{\prime}\right)=\int_{t_{0}}^{t_{1}} \sqrt{\rho^{2}+\rho^{\prime 2}} d t
$$

with the energy given by

$$
f\left(t, \rho, \rho^{\prime}\right)=\sqrt{\rho^{2}+\rho^{\prime 2}} .
$$

Obviously, (3.5) is the length of curve $\mathbf{z}$ between the two endpoints $\mathbf{z}\left(t_{0}\right)$ and $\mathbf{z}\left(t_{1}\right)$. It is wellknown that the Euler-Lagrange equation of (3.5) is given by

$$
\frac{\partial f}{\partial \rho}-\frac{d}{d t}\left(\frac{\partial f}{\partial \rho^{\prime}}\right)=0 .
$$

Now, we may derive from (3.6) and (3.7) that the Euler-Lagrange equation of (3.5) is the following second order differential equation:

$$
\rho \rho^{\prime \prime}-2 \rho^{\prime 2}-\rho^{2}=0 .
$$

After solving (3.8) we obtain $\rho=a \sec (t+b)$ for some real numbers $a, b$ with $a \neq 0$. Combining this with (3.3) gives

$$
\mathbf{z}(t)=a \sec (t+b) \mathbf{y}(t)
$$

Therefore, after applying Theorem 3 of [3], we conclude that the curve $\mathbf{z}(t)$ of (3.3) is a geodesic on the cone $\mathscr{C}$ if and only if $\mathbf{z}(t)$ is a rectifying curve. 


\section{An application}

A curve is the Euclidean 3 -space $\mathbb{E}^{3}$ is called planar if it lies in a plane in $\mathbb{E}^{3}$. From Theorem 1 we have the following.

Corollary 1. The only planar geodesics in a cone $\mathscr{C}$ in $\mathbb{E}^{3}$ are open portions of rulings.

Proof. It follows from the proof of Theorem 1 that if a geodesic on a cone does not lie in a ruling, then it must be a curve of the following form:

$$
\mathbf{z}(t)=a \sec (t+b) \mathbf{y}(t)
$$

where $a$ and $b$ are constants with $a \neq 0$ and $\mathbf{y}(t)$ is a unit speed curve lying in the unit sphere $S_{o}^{2}(1)$ centered at $o \in \mathbb{E}^{3}$.

Now, we claim that the curve $\mathbf{z}(t)$ has nonzero curvature $\kappa_{\mathbf{z}}$. Without loss of generality, we may assume $a=1$ and $b=0$. Thus (4.1) reduces to

$$
\mathbf{z}(t)=(\sec t) \mathbf{y}(t)
$$

From (4.2) we get

$$
\begin{aligned}
\mathbf{z}^{\prime} & =\sec (t)\left\{\mathbf{y}^{\prime}+(\tan t) \mathbf{y}\right\} \\
\mathbf{z}^{\prime \prime} & =(\sec t)\left\{\left(\sec ^{2} t+\tan ^{2} t\right) \mathbf{y}+2(\tan t) \mathbf{y}^{\prime}+\mathbf{y}^{\prime \prime}\right\}
\end{aligned}
$$

It is well-known that the curvature $\kappa_{\mathbf{z}}$ of $\mathbf{z}(t)$ is given by

$$
\kappa_{\mathbf{z}}=\frac{\left|\mathbf{z}^{\prime} \times \mathbf{z}^{\prime \prime}\right|}{\left|\mathbf{z}^{\prime}\right|^{3}}
$$

Thus $\kappa_{\mathbf{z}}=0$ holds if and only if $\mathbf{z}^{\prime} \times \mathbf{z}^{\prime \prime}=0$ holds. After combining this with (4.3) we conclude that $\kappa_{\mathbf{z}}=0$ holds if and only if we have

$$
\left\{\mathbf{y}^{\prime}+(\tan t) \mathbf{y}\right\} \times\left\{\left(\sec ^{2} t+\tan ^{2} t\right) \mathbf{y}+2(\tan t) \mathbf{y}^{\prime}+\mathbf{y}^{\prime \prime}\right\}=0
$$

On the other hand, because $\mathbf{z}(t)$ is a unit speed curve lying on $S_{o}^{2}(1)$, we know that $\kappa_{\mathbf{y}} \neq 0$ and $\mathbf{y}$ satisfies (cf. [8, page 34])

$$
\mathbf{y}=\left(\frac{1}{\kappa_{\mathbf{y}}}\right) \mathbf{n}_{y}+\left(\frac{1}{\kappa_{\mathbf{y}}}\right)^{\prime}\left(\frac{1}{\tau_{\mathbf{y}}}\right) \mathbf{b}_{y}
$$

where $\kappa_{\mathbf{y}}, \tau_{\mathbf{y}}, \mathbf{t}_{\mathbf{y}}, \mathbf{n}_{y}, \mathbf{b}_{y}$ are the curvature, the torsion, the unit tangent vector, the principal normal and the binormal of $\mathbf{y}$, respectively. Now, by substituting (4.5) into (4.4) and also by applying the Frenet formulas for $\mathbf{y}$, we find

$$
(\tan t) \kappa_{\mathbf{y}}\left(\frac{1}{\kappa_{\mathbf{y}}}\right)^{\prime}\left(\frac{1}{\tau_{\mathbf{y}}}\right) \mathbf{t}_{y}+\left(\frac{1}{\kappa_{\mathbf{y}}}\right)^{\prime}\left(\frac{1}{\tau_{\mathbf{y}}}\right) \mathbf{n}_{y}+\left(\kappa_{\mathbf{y}}-\frac{1}{\kappa_{y}}\right) \mathbf{b}_{\mathbf{y}}=0 .
$$


Since $\mathbf{t}_{y}, \mathbf{n}_{\mathbf{y}}, \mathbf{b}_{\mathbf{y}}$ are linearly independent, the coefficient of $\mathbf{b}_{\mathbf{y}}$ in (4.6) yields $\kappa_{\mathbf{y}}=1$. Hence (4.5) reduces to $\mathbf{y}=\mathbf{n}_{\mathbf{y}}$. So, after differentiating this equation with respect to the arc-length $t$ and using the Frenet formula, we find $\tau_{\mathbf{y}}=0$. Hence $\mathbf{y}$ is a planar curve with curvature $\kappa_{\mathbf{y}}=1$. Therefore $\mathbf{y}$ is a great circle of $S_{o}^{2}(1)$, which contradicts to our definition of cone. Thus $\kappa_{\mathbf{z}} \neq 0$, which shows the claim.

Since $\mathbf{y}$ and $\mathbf{y}^{\prime}$ are orthonormal vector fields, the first equation in (4.3) gives

$$
\left|\mathbf{z}^{\prime}\right|^{2}=\sec ^{4} t
$$

Thus (4.2), (4.3) and (4.7) imply that the normal component $\mathbf{z}^{N}$ of $\mathbf{z}$ satisfies

$$
\left\langle\mathbf{z}^{N}, \mathbf{z}^{N}\right\rangle=\langle\mathbf{z}, \mathbf{z}\rangle-\frac{\left\langle\mathbf{z}, \mathbf{z}^{\prime}\right\rangle^{2}}{\left|\mathbf{z}^{\prime}\right|^{2}}=1 .
$$

Hence we may conclude from Theorems 1 and 2 of [3] that $\mathbf{z}$ is rectifying with nonzero torsion. Consequently, $\mathbf{z}$ is never planar.

Remark 1 (Added on December 28, 2016). The author thanks Prof. Dong-Soo Kim who pointed out that rectifying curves had also been studied in [6].

\section{References}

[1] B.-Y. Chen, Geometry of submanifolds, Marcel Dekker, New York, 1973.

[2] B.-Y. Chen, Riemannian submanifolds, in: Handbook of Differential Geometry, Vol. 1 (2000), $187-418$.

[3] B.-Y. Chen, When does the position vector of a space curve always lie in its rectifying plane?, Amer. Math. Monthly, 110 (2003), 147-152.

[4] B.-Y. Chen, Differential Geometry of Warped Product Manifolds and Submanifolds, World Scientific, Hackensack, NJ, 2017.

[5] B.-Y. Chen and F. Dillen, Rectifying curves as centrodes and extremal curves, Bull. Inst. Math. Acad. Sinica 33 (2005), 77-90.

[6] D.-S. Kim, H.-S. Chung and K.-H. Cho, Space curves satisfying $\tau / \kappa=a s+b$, Honam Math. J., 15 (1993), 1-9.

[7] P. Lucas and J. A. Ortega-Yagues, Rectifying curves in the three-dimensional sphere, J. Math. Anal. Appl., 421 (2015), 1855-1868.

[8] R. S. Millman and G. D. Parker, Elements of Differential Geometry, Prentice-Hall Inc., NJ, 1977.

[9] W. Klingenberg, Lectures on Closed Geodesics, Springer-Verlag, Berlin-New York, 1978.

[10] J. Oprea, Differential Geometry and Its Applications, 2nd edition, The Mathematical Association of America, 2007.

[11] M. Spivak, A Comprehensive Introduction to Differential Geometry, vol. 2, Publish or Perish, Houston, TX, 1999.

Department of Mathematics, Michigan State University, 619 Red Cedar Road, East Lansing, Michigan 48824-1027, U.S.A.

E-mail: bychen@math.msu.edu 